\title{
A Double-Gaussian, Percentile-Based Method for Estimating Maximum Blood Flow Velocity
}

\author{
Caren Marzban, PhD, Paul R. Illian, BS, David Morison, BS, Pierre D. Mourad, PhD
}

Received February 20, 2013, from the Applied Physics Laboratory (C.M., P.R.I., D.M., P.D.M.) and Departments of Statistics (C.M.), Neurological Surgery (P.D.M.), and Bioengineering (P.D.M.), University of Washington, Seattle, Washington USA. Revision requested March 6, 2013. Revised manuscript accepted for publication April 15, 2013.

This work was supported by National Institutes of Health grant R43NS46824-01A1, National Space Biomedical Research Institute grant SMS00701-2009-513, and PhysioSonics, Inc (Bellevue, WA). Dr Mourad has a financial interest in PhysioSonics.

Address correspondence to Caren Marzban, PhD, Department of Statistics, University of Washington, Box 354322, Seattle, WA 981954322 USA.

E-mail:marzban@stat.washington.edu
Objectives - Transcranial Doppler sonography allows for the estimation of blood flow velocity, whose maximum value, especially at systole, is often of clinical interest. Given that observed values of flow velocity are subject to noise, a useful notion of "maximum" requires a criterion for separating the signal from the noise. All commonly used criteria produce a point estimate (ie, a single value) of maximum flow velocity at any time and therefore convey no information on the distribution or uncertainty of flow velocity. This limitation has clinical consequences especially for patients in vasospasm, whose largest flow velocities can be difficult to measure. Therefore, a method for estimating flow velocity and its uncertainty is desirable.

Methods - A gaussian mixture model is used to separate the noise from the signal distribution. The time series of a given percentile of the latter, then, provides a flow velocity envelope. This means of estimating the flow velocity envelope naturally allows for displaying several percentiles (eg, 95th and 99th), thereby conveying uncertainty in the highest flow velocity.

Results - Such envelopes were computed for 59 patients and were shown to provide reasonable and useful estimates of the largest flow velocities compared to a standard algorithm. Moreover, we found that the commonly used envelope was generally consistent with the 90th percentile of the signal distribution derived via the gaussian mixture model.

Conclusions - Separating the observed distribution of flow velocity into a noise component and a signal component, using a double-gaussian mixture model, allows for the percentiles of the latter to provide meaningful measures of the largest flow velocities and their uncertainty.

Key Words — blood flow; brain; head injury; noninvasive; transcranial Doppler sonography

$\mathrm{T}$ ranscranially derived Doppler measurements of blood flow in major cerebral arteries have found many clinical applications. ${ }^{1}$ In addition to assaying stroke risk due to sickle cell disease, dysfunction of cerebral autoregulation, and a patent foramen ovale, among other etiologies, some of the earliest applications targeted monitoring for vasospasm after subarachnoid hemorrhage. ${ }^{2-5}$ Cerebral vasospasm, the transient reduction of the diameter of 1 or more major cerebral arteries, can lead to reduced blood flow into the brain and hence cerebral ischemia and an increasing chance for neurologic damage. ${ }^{1}$ Monitoring for the onset of vasospasm remains an important application of transcranial Doppler sonography, with more 30,000 patients per year requiring daily monitoring for 1 week or more. ${ }^{6}$ Adding to this primarily 


\section{Author: Nonstandard abbreviations are written out, per journal style.}

civilian population are military patients with head injuries after exposure to explosions, typically roadside bombs, with half of these patients having cerebral vasospasm. ${ }^{7}$

Transcranial Doppler sonography measures the distribution of blood flow speeds within a blood vessel toward or away from the transducer, with negligible flow speeds adjacent to the blood vessel wall and maximum flow speeds near the center of the vessel. ${ }^{8}$ Critical for the assay of cerebral vasospasm, among other uses of transcranial Doppler sonography, is successful capture of the speed of the fastest flowing blood within the major cerebral arteries, since this value acts as a useful proxy for blood vessel narrowing. ${ }^{1}$ Capturing this speed is a particularly challenging problem, since vasospasm reduces the volume of blood flow while accelerating the blood flow speed, ${ }^{9}$ hence reducing the target for transcranial Doppler measurements while straining against the upper limits of ultrasound data processing due to aliasing. ${ }^{10}$

The time series of a flow velocity histogram is generally referred to as a spectrogram, and the time series of the maximum flow velocity is called an envelope. Although the spectrogram conveys a great deal of information, the envelope is often the only quantity a clinician examines. This practice is reasonable because the information contained in a spectrogram can be displayed in different ways, leading to different conclusions. For example, Figure 1 shows a spectrogram with 2 different color schemes; in the top panel, the spectrogram is linearly related to the color scale, whereas in the bottom panel, the colors are proportional to the square root of the spectrogram.

Figure 1. Spectrograms of 2 different mappings for assigning color to the flow velocity (FV) values: linear (top) and square root (bottom).
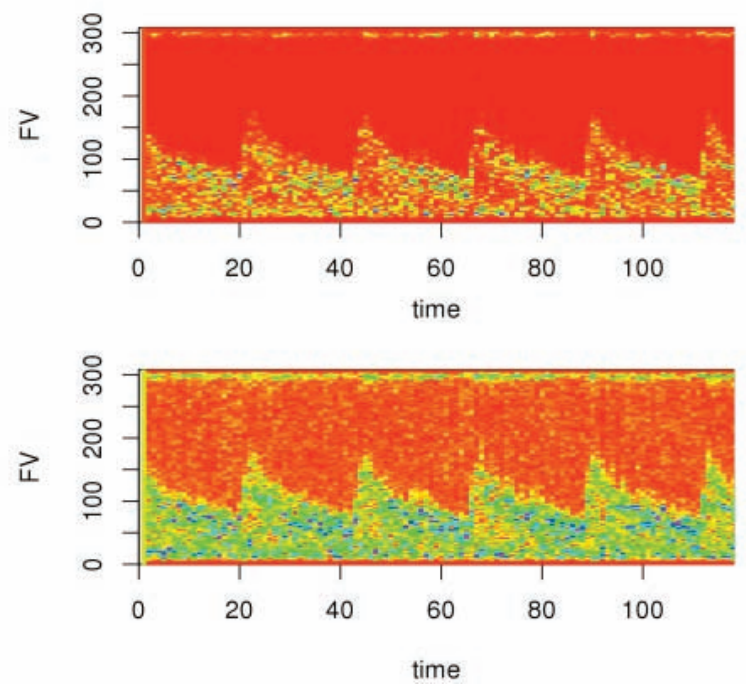

This inherent ambiguity in the information gleaned from a spectrogram also reflects itself in the corresponding envelope. In practice, observed flow velocity values can range from 1 to $300 \mathrm{~cm} / \mathrm{s}$. Therefore, to obtain a useful estimate of maximum flow velocity, one must introduce some criterion that defines what is meant by maximum. Many such criteria (standards for transcranial Doppler measurements ${ }^{11-15}$ ) are based on the cumulative histogram of flow velocity. Figure 2 shows an example of the relative frequency histogram of flow velocity (top) and the corresponding cumulative histogram (bottom) at a specific time for a specific patient (hereafter, patient $\mathrm{X}$ ); the latter is obtained by integrating the former from left to right. One may define the maximum flow velocity as the value at which the cumulative histogram "levels off," but there exist different criteria corresponding to different objective measures of that point. ${ }^{11-15}$ The vertical bar in Figure 2 marks the flow velocity at which it is maximum according to the modified geometric method. ${ }^{11,12}$ The top panel in Figure 3 shows the spectrogram and the modified geometric method envelope (in black) for patient $\mathrm{X}$.

Given that the proposed algorithm is compared with the modified geometric method algorithm, a brief review of the latter is in order. The modified geometric method algorithm and its variants ${ }^{11,12}$ effectively rotate (clockwise and about the origin) the cumulative histogram by some amount. The effect of such a rotation is that the point at which the cumulative histogram levels off translates to a point at which the rotated cumulative histogram reaches

Figure 2. Histograms of flow velocity (FV) for patient $X$ at a given instant in time (top), and the corresponding cumulative (ie, integrated) histogram (bottom).
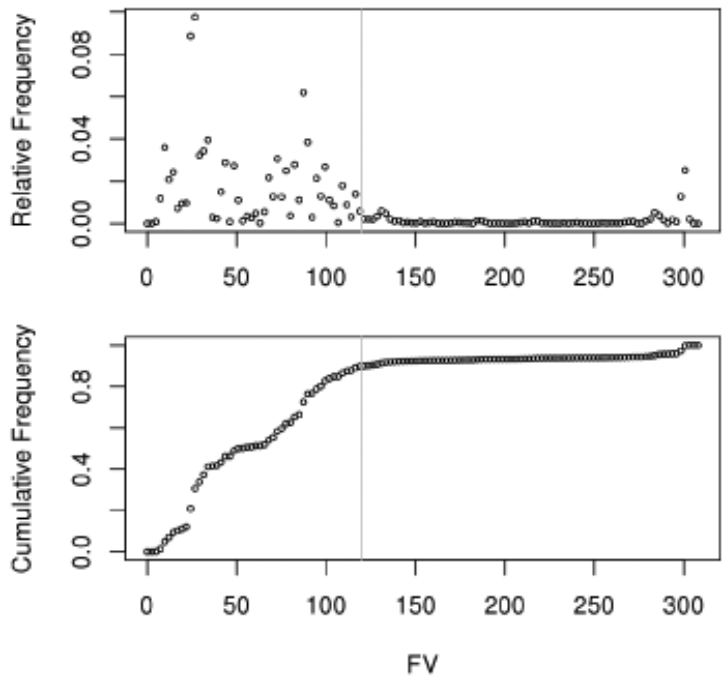
the maximum. Given certain smoothness constraints, it has been shown that this point corresponds to the maximum flow velocity. ${ }^{12}$ Note that the modified geometric method algorithm generates only point estimates of the maximum flow velocity and so provides no natural measure of uncertainty. This limitation is evident in the cumulative histogram in Figure 2: Although the modified geometric method provides an estimate of the point at which the cumulative histogram levels off, quantifying the corresponding uncertainty is by no means unambiguous. Conveying uncertainty is important because different levels of uncertainty call for different clinical actions.

The main proposals in this study were (1) to develop a new means of separating the noise and the signal components of a spectrogram and (2) to quantify the largest values in the signal using the concept of percentile. The $n$th percentile of a quantity $x$ is defined as the value of $x$ for which $n \%$ of the $x$ values are smaller. For example, the 95 th percentile of flow velocity is the flow velocity value at which $95 \%$ of flow velocity values are smaller. An envelope based on the 95th percentile of the histogram, then, provides a meaningful estimate of the "top 5\%" of flow velocities. Moreover, 2 (or more) envelopes at different percentiles can convey some notion of uncertainty. In this study, envelopes based on 3 percentiles were considered: 90th, 95th, and 99th.

Figure 3. Top, Spectrogram for patient X (color background) and 4 estimates of the envelope: modified geometric method envelope (black) and 90th, 95th, and 99th percentile-based envelopes. Bottom, Modified geometric method envelope (black), 95th percentile-based envelope (red), and 90th and 99th percentile-based envelopes (blue). FV indicates flow velocity.
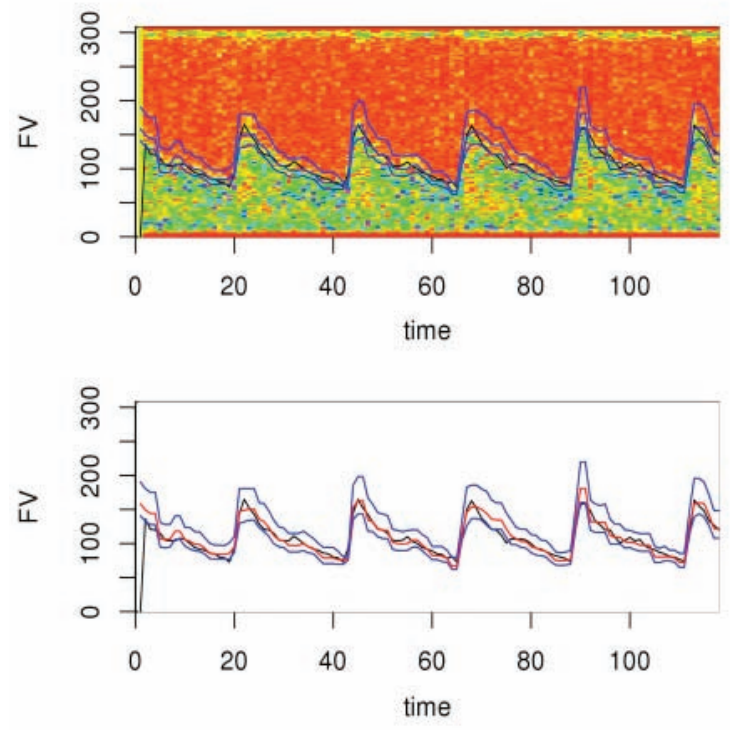

The use of a percentile to quantify the largest flow velocity presumes that the histogram is the correct histogram of flow velocity. In practice, observed spectrograms are contaminated by various types of noise. One of these sources of noise is evident in the small "hump" appearing on the right side of the (relative frequency) histogram in Figure 2. Flow velocity values and their associated percentiles are useful only if they pertain to the non-noise component of the histogram, hereafter called the signal distribution. In fact, the 90th percentile of the histogram shown in Figure 2 is near the right-most hump and therefore unrealistic. Therefore, to compute useful percentilebased envelopes, one must first disambiguate the signal and noise contributions to the histogram. To that end, here, a gaussian mixture model with 2 components ${ }^{16,17}$ was used to represent the noise and signal components, respectively. The component appearing to the left (closest to the origin) was defined as the signal distribution. Armed with the signal distribution, the 3 percentiles were computed at each time, and their time series was computed for 59 patients. The 3 percentile-based envelopes for patient $\mathrm{X}$ are shown in Figure 3, both with the spectrogram (top panel), and without (bottom panel).

In this article, the details of this percentile-based approach for estimating an envelope are presented. It was found that the estimates are visually consistent with the underlying spectrograms, and the modified geometric method envelope is approximately consistent with the 95th percentile envelope. Also, it is shown that percentilebased envelopes naturally allow for displaying envelope uncertainty.

\section{Materials and Methods}

\section{Data}

Patient data for this preclinical study were collected from a variety of hospitals in the United States, following a study at the University of Washington led by Dr Mourad. The data included arterial blood pressure and intracranial pressure, but those data were not used for this study; only sonographically derived transcranial Doppler spectra were used. Further details on the patient data may be found in an article by Marzban et al. ${ }^{18}$ In accordance with the Institutional Review Board for each hospital, informed consent was obtained from all patients or their families.

Doppler spectral time series of blood flow speed in the middle cerebral artery (or flow velocity - flow velocity) were acquired at each institution via clinically approved transcranial Doppler units. Data acquisition lengths varied from 5 to 30 minutes. All retrospective data processing and 
analysis were conducted at the Applied Physics Laboratory of the University of Washington. Doppler spectral time series data collected via the Applied Physics Laboratory's hospital cohort were initially digitized at $125 \mathrm{~Hz}$ in time and with a frequency resolution of $128 \mathrm{~Hz}$ at a given time. We then down-sampled these Doppler time series to a resolution of $40 \mathrm{~Hz}$ in time, sufficient to resolve all of the following features in all of our patients' data: systolic rise, diachrotic notch, and diastolic minimum. We also selected a fixed duration ( 118 time steps, or $\approx 3$ minutes) from each of the 59 patients for statistical analysis. This duration was sufficiently long to include several cardiac cycles while still allowing details of the envelopes to be visually evident.

\section{Data Processing and Statistical Analysis}

As mentioned in the introduction, the main aims of the proposed method were to first represent the instantaneous Doppler frequency distribution of the flow velocity at a given moment in time and then to produce spectral envelopes of those frequency distributions through time based on percentiles of that distribution in the Doppler frequency. Distinguishing the signal from the noise requires a model. In this article, it is assumed that the underlying distribution of flow velocity consists of 2 distributions, corresponding to the signal and noise, and that both are gaussian. This type of model is a special case of gaussian mixture models, wherein a distribution is assumed to be a linear combination of gaussian distributions. ${ }^{16,17}$ The weights in the linear combination (called mixing proportions) and the parameters of the gaussian distributions are then estimated from data via some optimization procedure. (In this study, the expectation-maximization algorithm was used, but other parameter estimation methods are equally adequate. The expectation-maximization algorithm maximizes the conditional expected log likelihood. ${ }^{19}$ ) Figure 4 shows the signal component (black) and the noise component (red) for the example shown in Figure 2. Also shown are the locations of the maximum flow velocity according to modified geometric method (black vertical line) and the 90th, 95th, and 99th percentiles of the signal distribution (blue vertical lines). All statistical analyses were performed in $\mathrm{R}^{20}$

\section{Results}

The previous section illustrates the proposed method on a given patient and at a given time. The top panel in Figure 3 shows the 3 percentile-based envelopes (blue curves) for patient X. It is evident that the percentile-based envelopes are consistent with the underlying spectrogram. The bot- tom panel in Figure 3 shows 4 envelopes; the modified geometric method envelope (black) is comparable with the 95th percentile envelope (red), sandwiched between the 90th and 99th percentile envelopes (blue). Clearly, displaying multiple percentile-based envelopes conveys a sense of the uncertainty in the maximum flow velocities.

Although technical, it is also worth mentioning 2 additional steps taken to generate the percentile-based envelopes shown in Figure 3: (1) The optimization algorithm used for the gaussian mixture model requires initial estimates of the parameters of interest. At time 0 , the initial values of the parameters are assigned randomly, and the optimization algorithm produces parameter values that characterize the 2 best-fit gaussian components of the flow velocity histogram at that time. At all "future" times, the initial values of the parameters are set to the final values obtained at the previous time step. This process allows some of the serial (auto) correlation in the spectrogram to be reflected in the envelopes. If the values of the gaussian mixture model parameters are initialized randomly for every and all time steps, the resulting envelopes are somewhat less smooth than those shown in Figure 3. (2) A runningmedian filter with a window size of 3 seconds is applied to the envelopes to smooth them even further. The size of the window (ie, 3 ) is obtained by trial and error and by a visual comparison with the modified geometric method envelope. The modified geometric method envelope too is smoothed by a variety of techniques, eg, an averaging (over multiple cardiac cycles) filter and a finite impulse response

Figure 4. Histogram of flow velocity (FV) for patient X (circles) and the estimated densities for the 2 gaussian components. The black vertical bar denotes the maximum flow velocity according to the modified geometric method, and the blue vertical bars correspond to the 90th, 95th, and 99th percentiles of the signal distribution on the left.

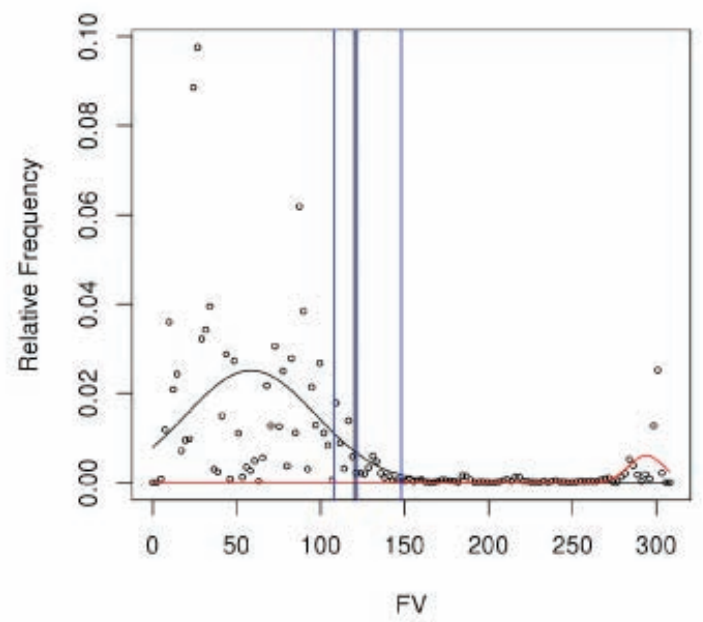




\section{Author: On Figure 5, please verify patient $X$ as changed from Spencer.}

filter. Although the smoothness of the displayed envelopes is important in a clinician's decision making, it is a feature that is easily controlled (eg, by a single parameter, such as the window size of the running-median filter) and so is not of serious concern.

The procedure was applied to all 59 patients in the data set. It is impractical to show all 59 figures analogous to Figure 3. Instead, the 3 percentile-based envelopes were compared with the modified geometric method envelope using scatterplots, which were then further summarized into scalar measures.

Each panel in Figure 5 shows the scatterplot of one of the percentile-based envelopes versus the modified geometric method envelope for patient $X$. There are 118 points on each scatterplot, corresponding to the 118 time steps displayed in Figure 3. The diagonal line is simply a line of slope $1, y$-intercept 0 . It is clear from the middle panel that the 95th percentile envelope generally agrees with the modified geometric method envelope. As expected, the 90th and 99th percentile envelopes are generally lower and higher, respectively, than the modified geometric method envelope. The disagreements between the percentile-based envelopes and the modified geometric method envelope are largest for higher flow velocity values. This result is especially evident in the 99th percentile envelope (bottom panel) in the manner in which the deviation of the scatterplot from the diagonal line is largest for higher flow velocity values. In other words, the systolic peaks of the 99th percentile envelope are in fact higher than might be expected from the modified geometric method envelope.

One may further summarize each of these panels by computing the root mean squared error between the modified geometric method envelope and each percentilebased envelope. For patient $X$, the root mean squared error values corresponding to the 3 panels in Figure 5 are 17.9, 16.9 , and $31.2 \mathrm{~cm} / \mathrm{s}$. In addition to confirming that the modified geometric method envelope is closest to the 95th percentile envelope (for patient X), these numbers have useful interpretations as well; eg, it can be said that the modified geometric method envelope and the 95th percentile envelope have a typical deviation of about 16.9 $\mathrm{cm} / \mathrm{s}$ across the 118 time steps examined.

Although useful for comparing different envelopes, root mean squared error values do not assess whether the difference in the envelopes is due to the amount of scatter in the scatterplot or to an overall shift. There exists a decomposition of root mean squared error that allows one to examine both contributions. The details of the decomposition are presented in "Appendix." Here, suffice it to say that the correlation coefficient (denoted
Corr) and bias (ie, mean [modified geometric method] mean [percentile-based method]) gauge the amount of scatter and the overall shift, respectively. The Corr values for the 3 panels in Figure 5 are 0.80, 0.81, and 0.81 (from top to bottom), suggesting that the amount of scatter in the 3 panels is comparable. Said differently, any of the 3 percentile-based envelopes provides an adequate approximation to the modified geometric method envelope, if/when correlation is the measure of agreement. The main difference between the 3 panels is in the Bias values: 8.5, 2.6 , and -23.5 (from top to bottom). These numbers have useful interpretations, too; eg, one can say that the 95th percentile envelope is generally shifted above the modified geometric method envelope by about $2.6 \mathrm{~cm} / \mathrm{s}$ for patient $\mathrm{X}$.

To compare the various envelopes across all of the patients, root mean squared error, Corr, and Bias were computed for all 59 patients. Their distributions are shown in Figure 6. The left-most box plot in the top panel summarizes the histogram (across 59 patients) of the root mean squared error computed for the 90th percentile envelope and the modified geometric method envelope (across 118 time steps).

The middle panel in Figure 6 is analogous to the top panel except that the measure of agreement between the modified geometric method envelope and the percentilebased envelope is Corr. The 3 box plots are comparable, implying that all 3 percentile-based envelopes are comparably correlated with the modified geometric method envelope across all 59 patients. The bottom panel shows that, on average (across all 59 patients), the 90th and 99th

Figure 5. Scatterplots of the 3 percentile-based envelopes and the modified geometric method envelope for patient X. FV indicates flow velocity.
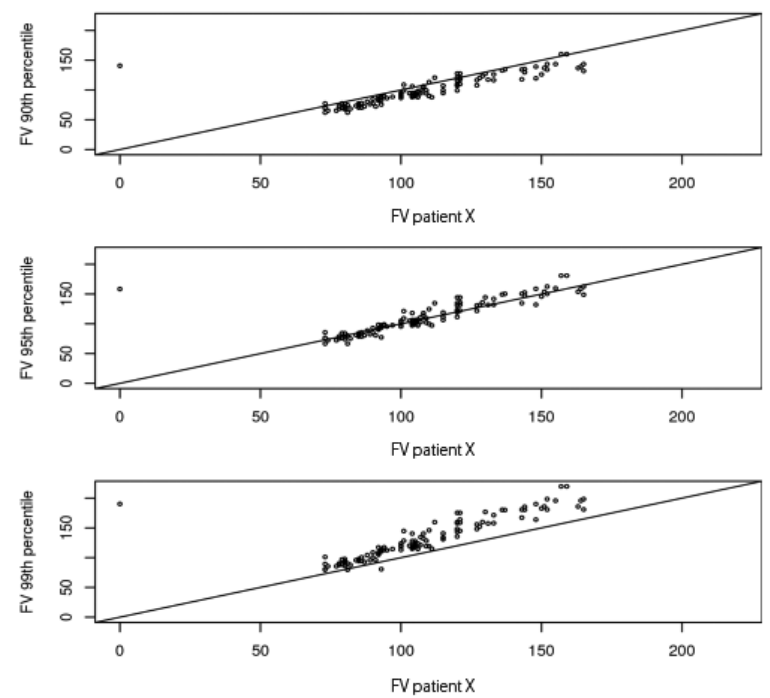
percentile envelopes are below and above the modified geometric method envelope, respectively. By comparing the 3 box plots in the top or bottom panel, it can be seen that the agreement between the modified geometric method envelope and the 95 th percentile envelope, noted for patient $X$, extends to all 59 patients.

It is worth displaying the envelope for a patient for whom such scalar measures are generally poor. Figure 7 shows the spectrogram and envelopes for the patient with the lowest Corr value. It is clear that the modified geometric method envelope (black) is in fact incorrect and that the percentile-based envelopes (blue) are more consistent with the underlying spectrogram. In other words, the low Corr value for this patient does not imply a defect on the part of the percentile-based envelope but rather a defect in the modified geometric method envelope.

\section{Discussion}

It has been shown that a double-gaussian mixture model can be used to identify a component of the histogram of flow velocity corresponding to a "signal." In turn, percentiles of this signal distribution provide envelopes, which meaningfully quantify the largest flow velocity. Not only are these percentile-based envelopes visually consistent with their underlying spectrograms, they are also objectively consistent with a commonly used envelope based on the modified geometric method. The percentile-based envelopes not only objectively quantify

Figure 6. Distribution of the root mean squared error (RMSE), correlation coefficient (Corr), and Bias (defined in "Appendix") for all 59 patients examined in this work.
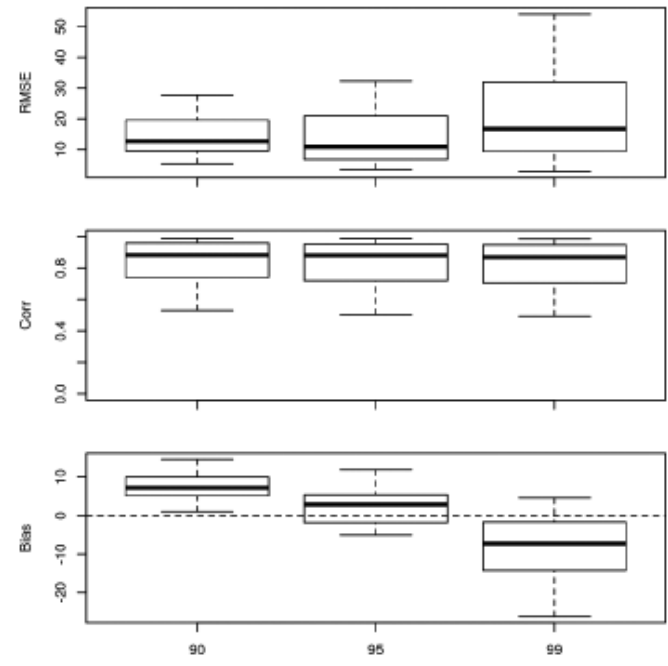

what is meant by "largest flow velocities" but also have the added advantage (over existing envelopes) of allowing for a natural display of uncertainty in the envelopes.

Given that the analysis is performed on real (not simulated) data, the true envelope is not known. As such, the quality of each envelope cannot be assessed objectively on its own merit. For that reason, the analysis here has been based on visually comparing an envelope with the underlying spectrogram (eg, as in Figure 3), or objectively comparing each of the percentile-based envelopes with the modified geometric method envelope (eg, Figures 5 and $6)$. In spite of the central role played by the modified geometric method envelope in comparing envelopes, it is important to recall that the modified geometric method envelope is not the true envelope (eg, see Figure 7). Here, it is used as a standard only because of its common usage.

Of the 3 percentile-based envelopes, the 95th percentile envelope is closest to the modified geometric method envelope, but the agreement is not perfect, as seen by the box plots in Figure 6. For example, according to the middle box plot in the bottom panel, on average (across patients), the 95th percentile envelope is slightly above the modified geometric method envelope. One may ask what is the exact percentile corresponding to the modified geometric method envelope, but the question itself is inappropriate because it elevates the status of the modified geometric method envelope to a "reference standard," when in fact it is not. What is more important is that the proposed approach produces a useful spectrogram as well

Figure 7. Same as Figure 3, but for a patient for whom the modified geometric method envelope (black) is clearly incorrect. FV indicates flow velocity.

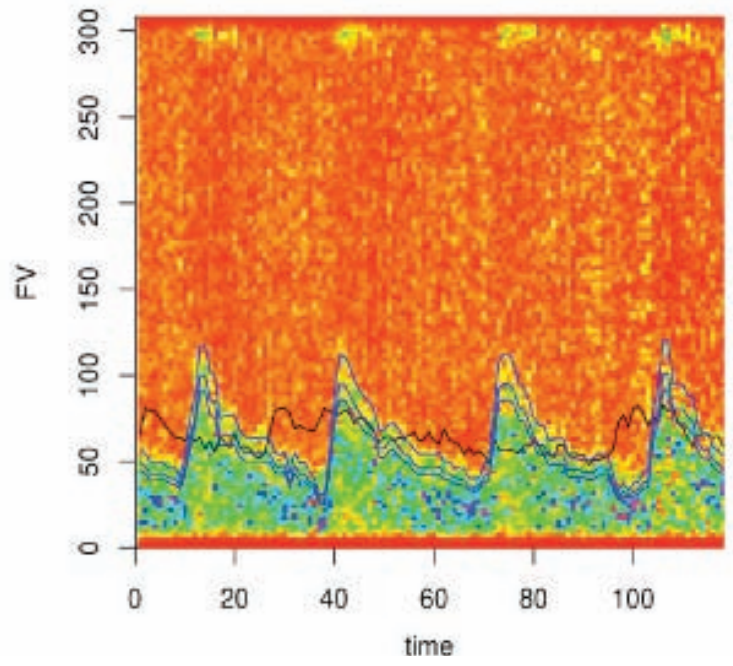


as an interpretable (in terms of percentiles) generalization of approaches that naturally produce only a single envelope (eg, modified geometric method).

A percentile-based envelope is based on a percentile of the signal distribution, in which the signal (and noise) distribution is defined from fitting a gaussian mixture model with 2 components to the whole histogram, at a given time. There is some justification for examining the 3 components. For example, in Figure 4, one may argue that there are possibly 3 overlaying histograms: 1 corresponding to the large hump on the left (ie, the signal), 1 associated with the small hump on the right (ie, associated with an aliasing reflection on Doppler imaging, and another corresponding to a uniform "background" spanning the full range of flow velocity values. We repeated the entire analysis but with 3 gaussian components. The results are inconclusive and require further research. For some patients, the results do not change substantially, but for others, they do in ways that can be considered "better" or "worse" depending on the measure of quality.

The percentiles of the signal distribution allow for meaningful envelopes, which can objectively assess the largest flow velocity. In addition, displaying multiple envelopes can convey information on the uncertainty in the observed flow velocity. One can envisage an alternative measure of uncertainty. For example, one may consider the envelope corresponding to a fixed percentile, say 95th, and then obtain the distribution of that quantity via some resampling method. ${ }^{21}$ In turn, that (sampling) distribution can be used to compute confidence intervals for the true 95th percentile envelope. Such interval estimates of the envelope can be useful in conveying uncertainty if/when a specific percentile is of interest. Otherwise, displaying multiple envelopes corresponding to different percentiles, as done here, is sufficient for conveying uncertainty.

\section{Appendix}

Let $x_{i}$ and $y_{i}$ denote the values of the modified geometric method envelope and a percentile-based envelope, respectively, at time $i$. The root mean square error between 2 envelopes is defined as the square root of the mean square error (MSE):

$$
M S E=\frac{1}{n-1} \sum\left(\mathrm{y}_{i}-\mathrm{x}_{i}\right)^{2},
$$

where $n$ is the length of the time steps (here, 118). It can be shown that

$$
M S E=s_{x}^{2}+s_{y}^{2}-2(\text { Corr }) s_{x y}+\frac{n}{n-1}(\text { Bias })^{2},
$$

where $s_{x}$ and $s_{y}$ are the sample standard deviations of $x$ and $y$, respectively, Corr denotes the Pearson correlation coefficient, and

$$
\text { Bias }=\text { mean } x-\text { mean } y .
$$

According to Equation 2, a comparison of 2 envelopes in terms of mean square error (or root mean square error) is equivalent to a comparison in terms of Corr and in terms of Bias; the former compares the correlation between the envelopes, and the latter measures the overall shift between them.

\section{References}

1. Sloan MA, Wozniak MA, Macko RF. Monitoring of vasospasm after subarachnoid hemorrhage. In: Babikian VL, Wechsler LR, Toole JF (eds). Transcranial Doppler Ultrasonography. Boston, MA: ButterworthHeinemann; 1999:109-128.

2. Aaslid R, Nornes H. Musical murmurs in human cerebral arteries after subarachnoid hemorrhage. J Neurosurg 1984; 60:32-36.

3. Aaslid R, Huber P, Nornes H. Evaluation of cerebrovascular spasm with transcranial Doppler ultrasound. J Neurosurg 1984; 60:37-41.

4. Aaslid R, Huber P, Nornes H. A transcranial Doppler method in the evaluation of cerebrovascular spasm. Neuroradiology 1986; 28:11-16.

5. Aaslid R. Transcranial Doppler assessment of cerebral vasospasm. Eur J Ultrasound 2002; 16:3-10.

6. Oertel M, Boscardin WJ, Obrist WD, et al. Posttraumatic vasospasm: the epidemiology, severity, and time course of an underestimated phenomenon - a prospective study performed in 299 patients. J Neurosurg 2005; 103:812-824.

7. Armonda RA, Bell RS, Vo AH, et al. Wartime traumatic cerebral vasospasm: recent review of combat casualties. Neurosurgery 2006; 59:1215-1225.

8. Babikian VL, Wechsler LR, Toole JF (eds). Transcranial Doppler Ultrasonography. Boston, MA: Butterworth-Heinemann; 1999.

9. Tsivgoulis G, Alexandrov AV, Sloan MA. Advances in transcranial Doppler Ultrasonogrphy. Curr Neurol Neurosci Rep 2009; 9(1):46-54

10. Tegeler $\mathrm{CH}$, Rotanakorn D. Physics and principles. In: Babikian VL, Wechsler LR, Toole JF (eds). Transcranial Doppler Ultrasonography. Boston, MA: Butterworth-Heinemann; 1999:3-11.

11. Moraes R, Adin N, Evans DH. The performance of three maximum frequency envelope detection algorithms for Doppler signals.J Vasc Invest $1995 ; 1: 126-134$.

12. Fernando KL, Mathews VJ, Clark EB. A mathematical basis for the application of the modified geometric method for maximum frequency estimation. IEEE Trans Biomed Eng 2004; 51:2085-2088.

13. Güler I,Hardalaç F, Kaymaz M. Comparison of FFT and adaptive ARMA methods in transcranial Doppler signals recorded from the cerebral vessels. Comput Biol Med 2002; 32:445-453. 
14. Östlund N, Yu J, Karlsson JS. Improved maximum frequency estimation with application to instantaneous mean frequency estimation of surface electromyography. IEEE Trans Biomed Eng 2004; 51:1541-1546

15. Rubin JM, Bude RO, Fowlkes JB, Spratt RS, Carson PL, Adler RS. Normalizing fractional moving blood volume estimates with power Doppler US: defining a stable intravascular point with the cumulative power distribution function. Radiology 1997; 205:757-765.

16. McLachlan GJ, Peel D. Finite Mixture Models. Hoboken, NJ: John Wiley \& Sons; 2000.

17. Bishop C. Pattern Recognition and Machine Learning. New York, NY: Springer; 2006.

18. Marzban C, Illian PR, Morison D, et al. A method for estimating zeroflow pressure and intracranial pressure. J Neurosurg Anesthesiol 2013; 25:25-32.

19. Dinov ID. Expectation maximization and mixture modeling tutorial. California Digital Library, Statistics Online Computational Resource website; December 9, 2008. http://repositories.cdlib.org/socr/ EM_MM.

20. R Development Core Team. R: a language and environment for statistical computing. R Project website; 2011. http://www.R-project.org.

21. Efron B, Tibshirani RJ. An Introduction to the Bootstrap. London, England: Chapman \& Hall; 1998. 